\title{
Integrative modeling of the pancreatic $\beta$-cell
}

Arthur Sherman

Laboratory of Biological Modeling, National Institute of Diabetes and Digestive and

Kidney Diseases, National Institutes of Health, Bethesda, MD USA

Richard Bertram

Department of Mathematics, Florida State University, Tallahassee, FL USA

Published in: Wiley Interscience Encyclopedia of Genetics, Genomics, Proteomics, and Bioinformatics, Part 3 Proteomics, M. Dunn, ed., Section 3.8 Systems Biology, R. L. Winslow, ed., John Wiley \& Sons, Ltd. DOI: 10.1002/047001153X.g308213

Keywords: insulin, diabetes, glucose, calcium, ion channels, endoplasmic reticulum, ATP, mitochondria, metabolism, acetylcholine, IP3

\begin{abstract}
:
Pancreatic $\beta$-cells secrete insulin, which regulates the concentration of plasma glucose. Dysfunction of this system is a necessary contributing factor to Type II diabetes, and may be sufficient. Insulin secretion is controlled by oscillations of membrane potential, called bursting oscillations, which drive oscillations of cytosolic calcium. We describe the development of mathematical models for this mechanism, beginning with the ChayKeizer model.
\end{abstract}

\section{Introduction}

Mammalian metabolism is well-adapted to switch between carbohydrate and lipid fuels depending on availability. One of the key hormones involved is insulin, which promotes glucose oxidation and fat storage when carbohydrate is abundant. In the developed world, unprecedented affluence has resulted in chronic excess of both nutrients relative to energy expenditure requirements, leading to an epidemic of obesity, 
hypertension, hyperlipidemia, and heart disease. An integral part of this metabolic syndrome is resistance to the effects of insulin, possibly as a compensation for overnutrition (Unger 2003). In response, the $\beta$-cells of the pancreatic islets of Langerhans over-secrete insulin to maintain plasma glucose within normal ranges. In many cases (currently about $6 \%$ of the population of the United States), the $\beta$-cells fail to compensate, leading to hyperglycemia (Type II diabetes), further morbidity, and premature death. For these reasons, there has been much interest in understanding how $\beta$-cells regulate plasma glucose. This control mechanism centers on complex patterns of oscillation in membrane potential and cytosolic calcium, which are difficult to understand without the aid of mathematical models. Those models are the focus of this review.

\section{Schematic model}

The $\beta$-cell models build on the Hodgkin-Huxley paradigm (Hodgkin and Huxley 1952), in which the plasma membrane is represented as an RC circuit, with the ion channels providing the resistance and the lipid bilayer providing the capacitance. The great success of such models in explaining the diverse electrical properties of neurons, muscle, and endocrine cells stems from the ability to reduce the electrical apparatus to a simple physical model, which can be represented by a small system of ordinary differential equations. The availability of dynamic readouts (voltage and calcium) with high time resolution has also been crucial. The validity of isolating the electrical module was strikingly demonstrated by transfecting voltage-dependent $\mathrm{Na}^{+}$and $\mathrm{K}^{+}$channels into non-excitable Chinese hamster ovary cells, which endowed them with neuron-like action potentials (Hsu and others 1993). 
The minimal parts list for the $\beta$-cell is shown in Fig. 1. The voltage-dependent $\mathrm{Ca}^{2+}$ channel conducts $\mathrm{Ca}^{2+}$ ions into the cell, which raises the transmembrane voltage, $V$, whereas the $\mathrm{K}^{+}$channel gates efflux of $\mathrm{K}^{+}$and restores $V$ to a low level. The temporal interaction of the two channels is sufficient to explain the repetitive spiking observed in $\beta$-cells. The $\mathrm{Ca}^{2+}$ influx also provides the primary chemical signal to trigger exocytosis of insulin-containing granules. These components are typical of many endocrine cells, but the $\beta$-cell needs an additional metabolic module to connect the level of electrical activity to plasma glucose concentration. Metabolism of glucose raises the ratio of ATP to ADP, which closes a third channel, the K(ATP) channel. Thus, in the absence of glucose, $\beta$-cells are electrically silent, but they generate $\mathrm{Ca}^{2+}$-dependent action potentials when glucose is elevated.

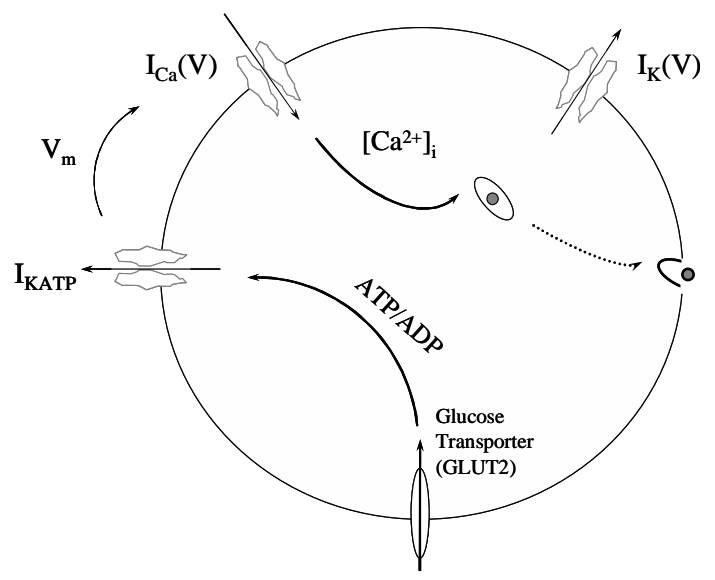

Fig. 1: Consensus schematic of $\beta$-cell. Glucose triggers depolarization and $\mathrm{Ca}^{2+}$ entry, which signals exocytosis of insulin granules.

This is the consensus schematic model for $\beta$-cells and is sufficient for understanding many aspects of diabetes pathology and therapy. For example, one of the 
classes of drugs used to treat Type II diabetes, the sulfonlyureas, block K(ATP) channels independent of glucose metabolism and augment insulin secretion (Ashcroft and Rorsman 1989). Also, recently it has been shown that either a surplus or a deficit of K(ATP) activity can lead to diabetes (Seino and Miki 2003). In the former case, K(ATP) channels that fail to close in response to glucose result in inadequate secretion of insulin In the latter case, K(ATP) channels that are always closed independent of glucose result in childhood hyperinsulinism. Some patients who survive this devastating hypoglycemia into adulthood become diabetic, possibly because their $\beta$-cells fail from chronic depolarization and exposure to high $\left[\mathrm{Ca}^{2+}\right]$ (Glaser 2003).

In both mice (Ashcroft and Rorsman 1989) and humans (Martin and Soria 1996), insulin secretion is controlled by oscillations of calcium, which are driven by bursts of action potentials with periods ranging from tens of seconds to several minutes (see Fig. 2 for an illustration using the model). We want to understand how bursting arises, how it is modulated by glucose and other signals, and how such a broad dynamic range is achieved. Three illustrative models are presented below in a didactic sequence of increasing complexity and explanatory power. Bear in mind that the actual historical development was more complex and subject to alternate interpretations.

\section{The Chay-Keizer Model}

The first widely-adopted mathematical model for bursting in $\beta$-cells was developed by Chay and Keizer (1983), based on the hypothesis of Atwater and Rojas that the slow dynamics of intracellular free $\mathrm{Ca}^{2+}$ are responsible for packaging impulses into bursts (Atwater and others 1980). That is, the sustained high voltage of the active phase 
of each burst would slowly increase cytosolic $\left[\mathrm{Ca}^{2+}\right](c)$, which would gradually activate a $\mathrm{Ca}^{2+}$-activated $\mathrm{K}^{+}(\mathrm{K}(\mathrm{Ca}))$ channel until a critical level was reached that would shut the spiking off. In the absence of spiking, $c$ would slowly recover, giving a long silent phase (Fig. 2).

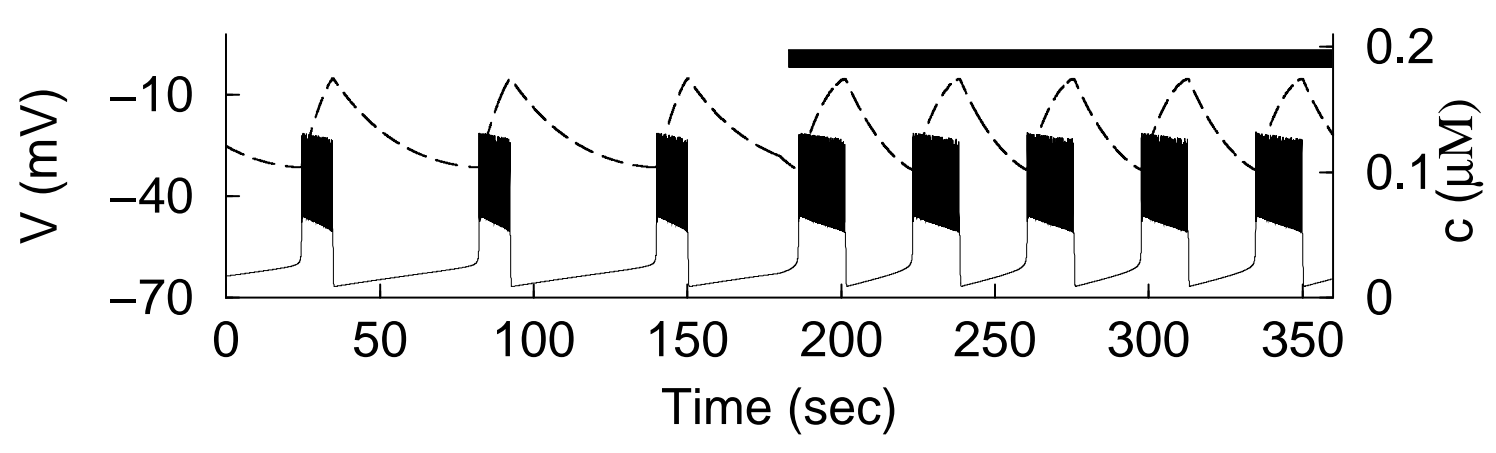

Fig. 2: simulation of glucose step with Chay-Keizer-like model (Equations 1-5).

Each plateau in voltage (V; solid)) is a train of brief spikes. Cytosolic calcium (c; dashed) rises slowly to terminate the bursts and recovers slowly in the silent phase. The black bar indicates period of elevated glucose.

Chay and Keizer also sought to explain how an increase in plasma glucose modulates the bursts by prolonging the active phases and shortening the silent phases, that is, increasing the plateau fraction. They suggested that increased glucose would increase activity of the plasma membrane $\mathrm{Ca}^{2+}$-ATPase (PMCA), slowing the rise of $c$ and accelerating its fall. In keeping with the modular approach, the machinery of metabolism enters only through its effect on the pump rate (black bar, Fig. 2).

The equations that correspond to the above scheme and that were used to produce Fig. 2 are in outline: 


$$
\begin{gathered}
\frac{d V}{d t}=-\left[I_{C a}(V)+I_{K}(V, n)+I_{K(C a)}(V, c)+I_{K(A T P)}(V)\right] / C_{m} \\
\frac{d n}{d t}=\left[n_{\infty}(V)-n\right] / \tau_{n} \\
\frac{d c}{d t}=f_{c y t} J_{m e m} .
\end{gathered}
$$

(The full equations and parameter values are posted at http://mrb.niddk.nih.gov/sherman.) The electric circuit is represented by equations 1 and 2 for the membrane potential $(V)$ and the activation level (open probability) of the voltage-dependent $\mathrm{K}^{+}$current $(n)$, respectively. Equation 1 is Kirchhoff's law, which balances capacitive current with the four ionic currents, the voltage-dependent $\mathrm{Ca}^{2+}\left(I_{C a}\right)$, voltage-dependent $\mathrm{K}^{+}\left(I_{K}\right), \mathrm{Ca}^{2+}-$ dependent $\mathrm{K}^{+}\left(I_{K(C a)}\right)$, and ATP-sensitive $\mathrm{K}^{+}\left(I_{K(A T P)}\right)$ currents. Note that in order to obtain spikes, the RC circuit must be elaborated by assuming that the resistive elements (i.e. the ion channels) change their conducting state in response to $V$. These changes have been measured (Ashcroft and Rorsman 1989) and incorporated semi-quantitatively into the model. For the present, the conductance of $I_{K(A T P)}$ is held constant, unlike the conductances of the other currents, which vary as $V$ or $c$ change in time. Later we will introduce dependence on glucose through the ATP production rate.

The slow dynamics of $c$ are modeled by simple mass balance, assuming one wellmixed compartment. Influx through $\mathrm{Ca}^{2+}$ channels increases $c$ while efflux through the PMCA decreases it:

$$
J_{m e m}=-\alpha I_{C a}(V)-k_{P M C A} c
$$

(Note that $I_{C a}$ is negative by convention, so $-\alpha I_{C a}$ is positive.) The net flux of $\mathrm{Ca}^{2+}$, $J_{m e m}$, is multiplied by $f_{c y t}$, the ratio of free to total (free + buffered) $\mathrm{Ca}^{2+} \cdot f_{c y t}$ is small here 
(0.00025), which makes $c$ slow. Calcium responds to the membrane potential through $I_{C a}$, and in turn influences the membrane potential through $I_{K(\mathrm{Ca})}=g_{K(\mathrm{Ca})} \omega\left(V-V_{K}\right)$, where the activation variable $\omega$ is:

$$
\omega=\frac{c^{2}}{c^{2}+K_{D}^{2}}
$$

A noteworthy feature of the Chay-Keizer model is that the voltage plateau is not explicitly included in the model. It is an emergent property arising from the $V$ - $n$ interactions. This contrasts with an earlier model (Matthews and O'Connor 1979), which postulated a special plateau current. This made for a more complex and cumbersome model than Chay-Keizer and may have contributed to its later neglect, in spite of its anticipation of many aspects of Chay-Keizer and other later models.

\section{The Endoplasmic Reticulum: A Second $\mathrm{Ca}^{2+}$ Compartment}

In addition to accounting for two major known properties of $\beta$-cells, the ChayKeizer model made a prediction that cytosolic $\mathrm{Ca}^{2+}$ would oscillate, with a slow rise and fall. When $\mathrm{Ca}^{2+}$ was later imaged in $\beta$-cells (Valdeolmillos and others 1989), it was indeed seen to rise and fall, which was not surprising, but the kinetics were not as expected: $c$ rose rapidly at the beginning of the active phase to a plateau and sometimes even fell slightly towards the end of the active phase. The $\mathrm{K}(\mathrm{Ca})$ channel hypothesis fell into disfavor, but has been revived, in part because of the discovery of a candidate $\mathrm{K}(\mathrm{Ca})$ channel in $\beta$-cells (Goforth and others 2002; Göpel and others 1999), and in part because of an improved model due to Chay (1996), which included a second, internal $\mathrm{Ca}^{2+}$ compartment, representing the endoplasmic reticulum (ER). 
There are several reasons for including the ER. As a storehouse for $\mathrm{Ca}^{2+}$ it can influence the $\mathrm{Ca}^{2+}$ dynamics of both excitable and non-excitable cells (Berridge 1997). In islets in particular, releasing $\mathrm{Ca}^{2+}$ from the ER by either blockade of the $\mathrm{ER}^{2}{ }^{2+}$ pump (SERCA) or activation of $\mathrm{IP}_{3}$ receptors dramatically affects electrical activity, $\mathrm{Ca}^{2+}$ oscillations, and secretion (Bertram and others 1995b; Goforth and others 2002; Worley and others 1994).

The ER compartment is incorporated into the model by adding an ER-to-cytosol flux term $\left(J_{e r}\right)$ to equation (3) and by adding a differential equation for the free $\mathrm{ER} \mathrm{Ca}^{2+}$ concentration $\left(c_{e r}\right)$. All forms of efflux are subsumed in a leakage pathway $\left(J_{\text {leak }}\right)$, and influx occurs through SERCA pumps $\left(J_{S E R C A}\right)$ :

$$
J_{e r}=J_{\text {leak }}-J_{\text {SERCA }} .
$$

We neglect the known non-linearities of these processes and write $J_{\text {leak }}=p_{\text {leak }}\left(c_{e r}-c\right)$ and $J_{\text {SERCA }}=k_{\text {SERCA }} c$. The effect of $\mathrm{IP}_{3}$ can be represented simply by an increase in $p_{\text {leak. }}$. The equations for $c$ and $c_{e r}$ are:

$$
\begin{aligned}
& \frac{d c}{d t}=f_{c y t}\left(J_{m e m}+J_{e r}\right) \\
& \frac{d c_{e r}}{d t}=-f_{e r}\left(V_{c y t} / V_{e r}\right) J_{e r},
\end{aligned}
$$

where $f_{e r}$ is the ER $\mathrm{Ca}^{2+}$ buffering factor, and $V_{c y t}$ and $V_{e r}$ are the cytosolic and ER volumes. 


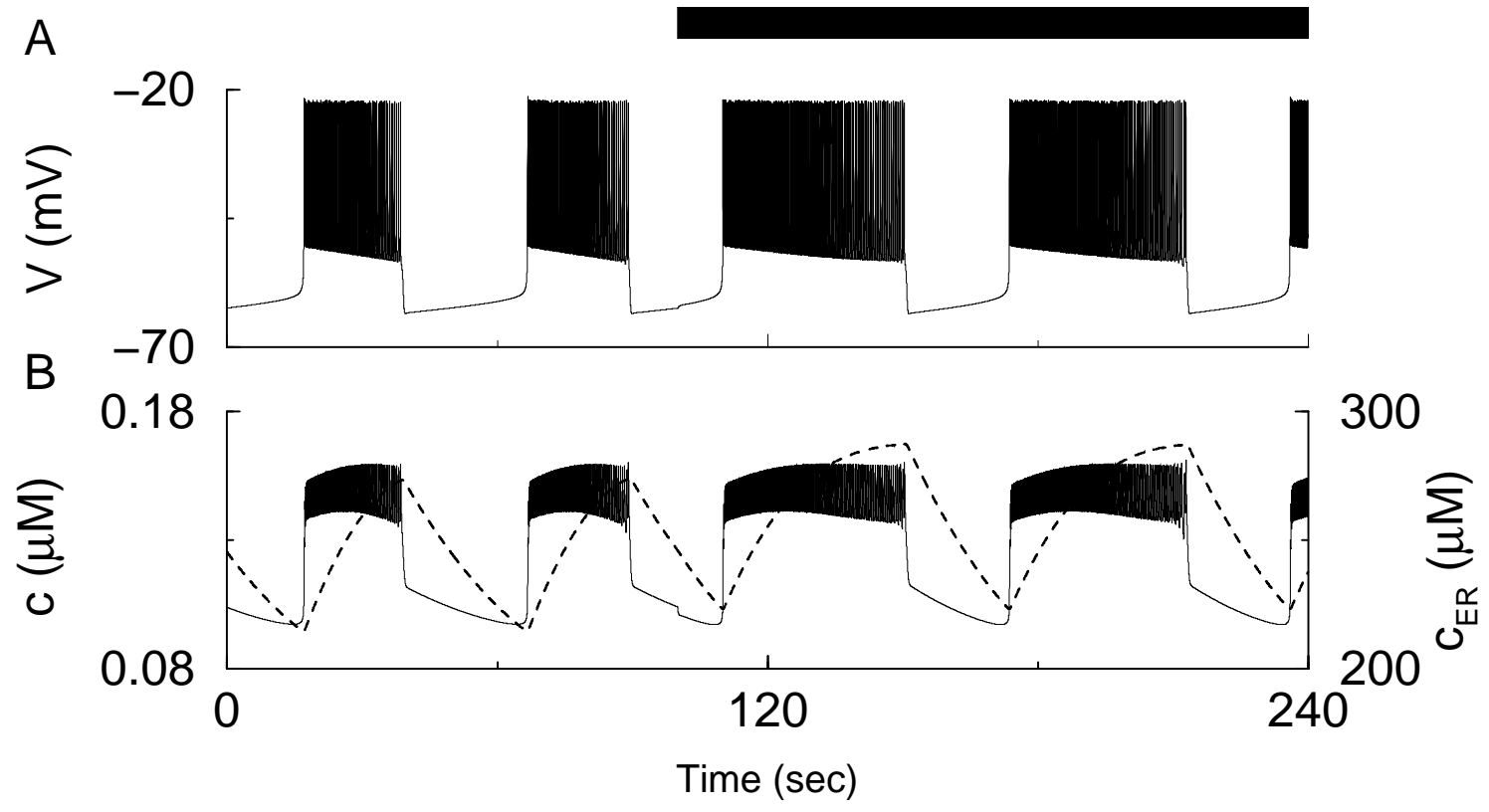

Fig. 3: Chay-Keizer model with a slow ER compartment ( $c_{E R}$, dashed). c now has fast and slow components, and increasing PMCA rate now increases both plateau fraction and mean $c$.

The behavior of the enhanced model is shown in Fig. 3. The rapid jump in $c$ at the beginning of each active phase reflects the intrinsic kinetics of cytosolic $\mathrm{Ca}^{2+}$, which have been accelerated compared to Fig. 2 by reducing the cytosolic buffer capacity (i.e. increasing $f_{c y t}$ to the more realistic value of 0.01 ). This rise in $c$ does not alone provide adequate inhibition to terminate the active phase, but as the ER slowly fills, $c$ is pushed up to the necessary critical level. When spiking ceases, $c$ jumps down rapidly and then slowly decays as the ER gives back the $\mathrm{Ca}^{2+}$ it took in during the active phase. Thus, like a buffer, the ER slows down $c$, but because the ER is slow (whereas buffers are fast), $c$ displays two distinct kinetic phases, in agreement with experiments. 
The model parameters in Fig. 3 are chosen to give an oscillation period comparable to that typically observed in islets $(10-60 \mathrm{sec})$. However, if $g_{K(\mathrm{Ca})}$ is increased, the initial jump in $c$ becomes sufficient to terminate an active phase without assistance from $c_{E R}$, resulting in a period of only a few seconds. (Similar fast bursting can also be obtained by partially emptying the ER. See Fig. 4.) Conversely, reducing $g_{K(\mathrm{Ca})}$ (and possibly adjusting $k_{P M C A}$ ), produces periods of several minutes, which are also observed in experiments. This broad range of frequencies is possible because the time scale of oscillations is determined by mixing the relatively fast $c$ and the relatively slow $c_{E R}$ components. We call this "phantom bursting" because the time scale is not determined by any single process (Bertram and Sherman 2004a).

A comparison of Figs. 2 and 3 shows that the ER enhances the model in another way. Whereas both the original Chay-Keizer model and the ER model explain how glucose can raise plateau fraction, in the one-compartment Chay-Keizer model, this does not lead to an increase in the mean level of cytosolic $\left[\mathrm{Ca}^{2+}\right]$. In the ER model, in contrast, the approximately square shape of $c$ means that an increase in plateau fraction produces an increase in the average level of $c$, without any change in the minimum and maximum values, as more time is spent at the higher level relative to the lower level. Note that this occurs in spite of the fact that the rate of pumping $\mathrm{Ca}^{2+}$ out of the cell is increased, illustrating that steady-state intuitions may not apply when there are oscillations.

As alluded to earlier, inclusion of the ER also allows the model to account for the effects of acetylcholine (ACh) application (Fig. 4). In vivo, ACh is released from parasympathetic nerve terminals in the pancreas to stimulate the islets in response to food 
ingestion (Woods and Porte 1974). This auxiliary control mechanism raises insulin in anticipation of the coming glucose load and helps limit the rise in plasma glucose. At the cell level, ACh stimulates the production of $\mathrm{IP}_{3}$, which activates $\mathrm{IP}_{3}$ receptors on the ER. Here we again collapse an external regulatory module into a change in a parameter of the core electrical/calcium module: the efflux permeability $p_{\text {leak }}$ is stepped up 45 -fold. This is sufficient to produce the increase in burst frequency seen experimentally. However, in order to capture the rise in $V$ and $c$ that are also seen, we need to add a small depolarizing current $\left(I_{A C h}=g_{A c h}\left(V-V_{A C h}\right)\right.$, where $g_{A C h}=5 \mathrm{pS}$ and $\left.V_{A C h}=0\right)$ to the $V$ equation. Such a current has now been found (Mears and Zimliki 2004; Rolland and others 2002). The depolarized bursting and elevation of the cytosolic $\mathrm{Ca}^{2+}$ concentration are critical because they contribute to the increase in insulin secretion.
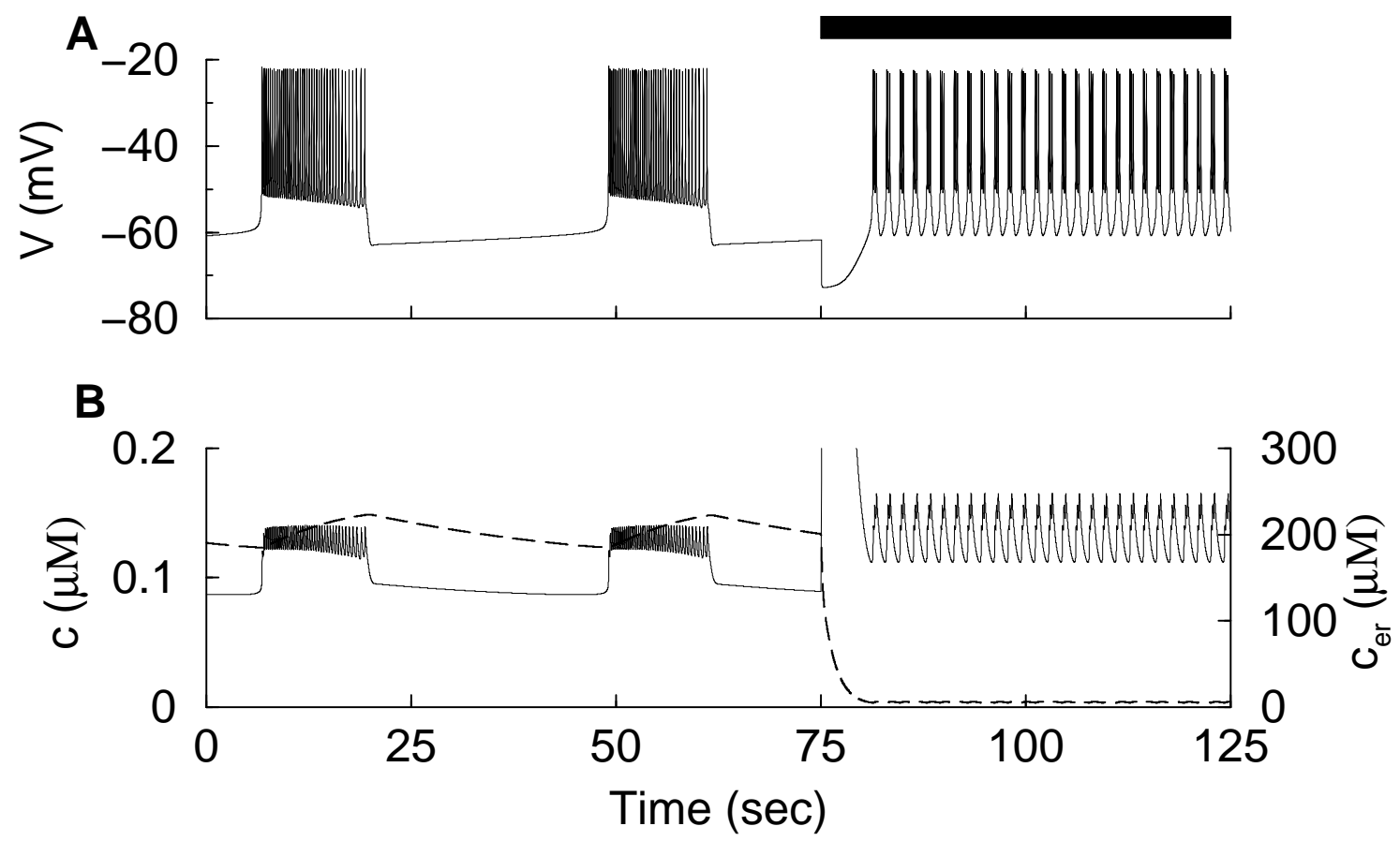

Fig. 4: Response of model with ER to acetylcholine (ACh), modeled as a step increase in $p_{l e a k}$, throughout period denoted by black bar. Release of $\mathrm{Ca}^{2+}$ from $\mathrm{ER}(\mathrm{B}$; 
dashed) gives a transient sharp peak of c (solid; truncated for clarity). Plateau of high c is maintained by an ACh-activated inward current, which drives depolarized, fast bursting $(A)$.

\section{Oscillations in glucose metabolism}

We now consider the glucose dependence of the K(ATP) channel. If the K(ATP) conductance is assumed to decrease with external glucose concentration, then plateau fraction is modulated similarly to the changes obtained in Figs. 1 and 2 by varying PMCA pump rate (not shown). Beyond this, there is some evidence that ATP and ADP concentrations in $\beta$-cells vary over time (Ainscow and Rutter 2002) in a $\mathrm{Ca}^{2+}$-dependent manner (Kennedy and others 2002). This was proposed by Keizer and Magnus (Keizer and Magnus 1989), who assumed that the rate of ATP production decreases when cytosolic $\mathrm{Ca}^{2+}$ inhibits the mitochondrial membrane potential. Phenomenologically, this can be represented by the following equation for ADP concentration:

$$
\frac{d[A D P]}{d t}=k_{a}\left[A T P-A D P \exp \left(r\left(1-c / r_{1}\right)\right)\right]
$$

We assume that the total nucleotide concentration, $A D P+A T P$, is constant, so no differential equation is needed for ATP. The effect of ATP to block the K(ATP) conductance is antagonized by ADP by competitive inhibition:

$$
I_{K(A T P)}=g_{K(A T P)} \frac{1+A D P / K_{1}}{1+A D P / K_{1}+A T P / K_{2}}\left(V-V_{K}\right)
$$

The net result is that a rise in $c$ results in a slow (minutes) rise in $A D P$ and an increase in $I_{K(A T P)}$. An increase in glucose can now be represented by an increase in the parameter $r$ 
in Equation 10 in combination with changes in pump rates. We illustrate this in Fig. 5 for the situation where glucose is raised from a sub-threshold level to a level that permits bursting. Low glucose is simulated by reducing $r$ and SERCA pump rate, $k_{S E R C A}$, which drains the ER and elevates $A D P$. Starting in that condition, we assume that when glucose is raised, $k_{\text {SERCA }}$ increases rapidly, producing a transient drop in $c$ (Fig. 5B). Over several minutes $A D P$ declines, reducing $\mathrm{K}(\mathrm{ATP})$ conductance, and $V$ gradually increases. When the voltage threshold for activation of $I_{C a}$ is reached, $V$ jumps up sharply and spiking begins. The ER, however, is still depleted and acts as a sink for $\mathrm{Ca}^{2+}$. This limits activation of the $\mathrm{K}(\mathrm{Ca})$ conductance, resulting in a prolonged period of spiking. After several more minutes, the ER is filled, and bursting can begin. $A D P$ oscillates during the bursting phase (inset, Fig. 5D) and combines with $c$ to drive the bursting. Thus, with inclusion of dynamics for $A D P$, the model is able to account for the complex tri-phasic transient of electrical and cytosolic $\mathrm{Ca}^{2+}$ activity observed experimentally (Bertram and others 1995b; Goforth and others 2002; Worley and others 1994). 

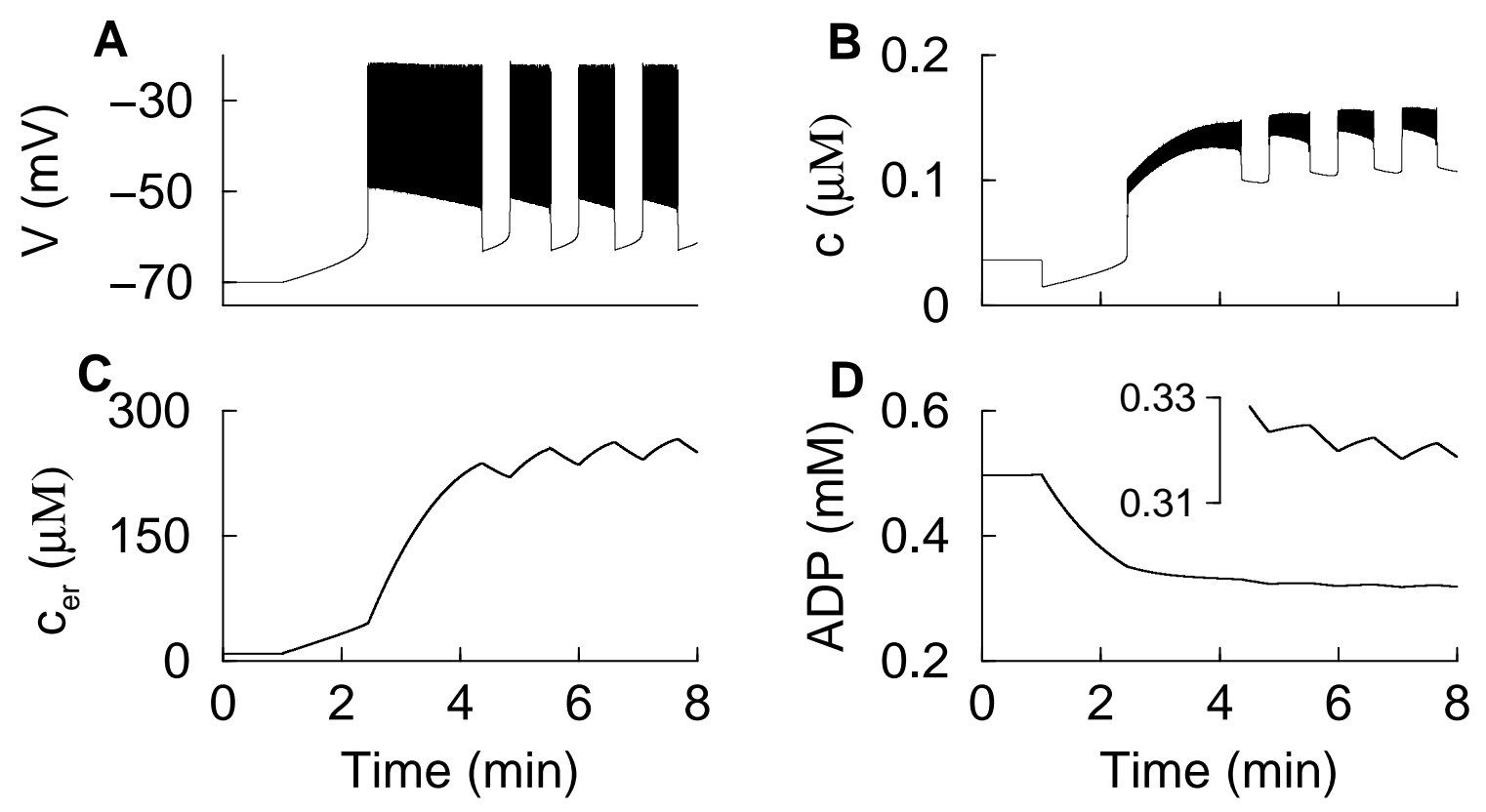

Fig. 5: Model with ER and calcium feedback on nucleotide dynamics. Glucose is stepped at $T=1$ min, initiating a tri-phasic transient (see text). In the steady state, ADP oscillates as well as cytosolic and ER calcium.

The incorporation of two very slow processes, $c_{E R}$ and $A D P$, makes the model more robust and endows it with some redundancy. This may help account for the observations that oscillations can persist when the ER is disabled by blocking SERCA (Fridlyand and others 2003) (K(ATP) can compensate for the loss of $\mathrm{K}(\mathrm{Ca})$ ) or when K(ATP) channels are blocked by sulfonylureas (Rosario and others 1993) (K(Ca) can compensate for the loss of K(ATP)). (See alsoBertram and Sherman 2004b). A third slow process recently proposed by Fridlyand et al (2003), $\mathrm{Na}^{+}$accumulation that activates the $\mathrm{Na}^{+}$pump, might also be helpful to explain this complex phenomenology. 


\section{Summary and Prospects}

We have traced in outline the evolution of the Chay-Keizer family of models over 20 years. The first models explained bursting of membrane potential and modulation of plateau fraction by glucose. They also predicted oscillations of cytosolic $\mathrm{Ca}^{2+}$, which were later seen, but with a different kinetic character. This led to a second wave of models with more accurate $\mathrm{Ca}^{2+}$ kinetics, and predictions of oscillations in $\mathrm{ER}^{2+}$. The latter have not yet been demonstrated experimentally, and indeed their existence has been challenged based on ER measurements in permeabilized cells (Tengholm and others 2001). Measurements of $\mathrm{ER} \mathrm{Ca}^{2+}$ in intact cells undergoing cytosolic $\mathrm{Ca}^{2+}$ oscillations are needed to test this prediction.

The current generation of models can account for the full range of observed oscillation periods, spanning two orders of magnitude, from seconds to minutes. They also account for modulation of electrical activity (and, implicitly, insulin secretion) by cholinergic stimuli as well as glucose (Fig. 4), and the complex transients seen when glucose is first elevated (Fig. 5). The initial prolonged spiking phase may be related to the early large peak of insulin secretion, which is lost in Type II diabetes (Kahn 2001). However, a serious treatment of secretion will require consideration of the dynamics of vesicle exocytosis and recycling (Rorsman and Renstrom 2003) in response to both local (sub-membrane) and bulk cytosolic $\mathrm{Ca}^{2+}$ and also to non- $\mathrm{Ca}^{2+}$ signals.

Future models will need to address other modulators, such as glucagon-like peptide, which potentiates secretion by elevating cAMP, and epinephrine, which blocks secretion and slows down oscillations. The former pathway is a drug target of great 
current interest because, unlike K(ATP) channel blockade, it is only effective when glucose is elevated, eliminating the danger of therapy-induced hypoglycemia. Recent data indicate that insulin may have both stimulatory and inhibitory effects on its own secretion (Khan and others 2001). This is an unsettled area that merits further investigation both experimentally and theoretically.

We have included metabolic oscillations based on $\mathrm{Ca}^{2+}$-dependent effects in the mitochondria (Fig. 5). However, there is also some evidence for glycolytic oscillations (Tornheim 1997). One possible role of glycolytic oscillations is in explaining the packaging of the bursts themselves into higher-order patterns, or "bursts of bursts" (Bertram and others 2004; Wierschem and Bertram 2004), and other roles are currently under investigation.

For brevity, we have described here only models for a single cell, representing an electrically synchronized islet. For an overview of models that explicitly treat the nonobvious effects of electrical coupling see Sherman (1996).

Thanks to judicious pruning of unnecessary detail, the current models are similar in complexity to the original Chay-Keizer model, in spite of their greater explanatory power. The use of simple models has paid dividends in terms of insight and mathematical analysis that goes beyond mere simulation. Of particular note is the establishment of a general classification scheme for burst mechanisms (Bertram and others 1995a). This has not only enhanced our understanding of the $\beta$-cell models, but also situated them in a general perspective of bursting models for neurons and other secretory cells. 
The $\beta$-cell has provided an interesting case study of the interaction of physiology and modeling with genomic methods. For example, the K(ATP) channel was identified by physiological experiments, and its possible roles, as either a passive modulator or active participant in oscillations, have been clarified by modeling. In parallel, sequencing has suggested how its structure underlies its function, and genetic scans have uncovered connections to disease (hyperinsulinism and Type II diabetes). Yet, we do not yet know why in rats glucose does not modulate plateau fraction, but rather gross depolarization (Antunes and others 2000), similarly to ACh in mice (Fig. 4). The islet $\alpha$ cells in both species also possess K(ATP) channels, but their response is just the reverse of $\beta$-cells - electrical activity and secretion are inhibited by glucose. How such different behaviors can arise in cell types that differ in rather subtle and quantitative ways poses a challenge for future studies integrating all the techniques at our disposal.

\section{References}

Ainscow EK, Rutter GA. 2002. Glucose-stimulated oscillations in free cytosolic ATP concentration imaged in single islet $\beta$-cells. Diabetes 51:S162-S170.

Antunes CM, Salgado AP, Rosario LM, Santos RM. 2000. Differential patterns of glucose-induced electrical activity and intracellular calcium responses in single mouse and rat pancreatic islets. Diabetes 49(12):2028-38.

Ashcroft FM, Rorsman P. 1989. Electrophysiology of the pancreatic $\beta$-cell. Prog. Biophys. Mol. Biol. 54:87-143.

Atwater I, Dawson CM, Scott A, Eddlestone G, Rojas E. 1980. The nature of the oscillatory behavior in electrical activity for the pancreatic $\beta$-cell. In: Thieme G, 
editor. Biochemistry and Biophysics of the Pancreatic $\beta$-cell. New York: Verlag. p 100-107.

Berridge MJ. 1997. Elementary and global aspects of calcium signalling. J. Physiol. 499:291-306.

Bertram R, Butte M, Kiemel T, Sherman A. 1995a. Topological and phenomenological classification of bursting oscillations. Bull. Math. Biol. 57:413-439.

Bertram R, Satin L, Zhang M, Smolen P, Sherman A. 2004. Calcium and glycolysis mediate multiple bursting modes in pancreatic islets. Biophys J 87(5):3074-87.

Bertram R, Sherman A. 2004. A calcium-based phantom bursting model for pancreatic islets. Bull Math Biol 66(5):1313-44.

Bertram R, Smolen P, Sherman A, Mears D, Atwater I, Martin F, Soria B. 1995b. A role for calcium release-activated current (CRAC) in cholinergic modulation of electrical activity in pancreatic $\beta$-cells. Biophys. J. 68:2323-2332.

Chay TR. 1996. Electrical bursting and luminal calcium oscillation in excitable cell models. Biol. Cybern. 75:419-431.

Chay TR, Keizer J. 1983. Minimal model for membrane oscillations in the pancreatic $\beta$ cell. Biophys. J. 42:181-190.

Fridlyand LE, Tamarina N, Philipson LH. 2003. Modeling of Ca2+ flux in pancreatic beta-cells: role of the plasma membrane and intracellular stores. Am J Physiol Endocrinol Metab 285(1):E138-54.

Glaser B. 2003. Dominant SUR1 mutation causing autosomal dominant type 2 diabetes. Lancet 361(9354):272-3. 
Goforth PB, Bertram R, Khan FA, Zhang M, Sherman A, Satin LS. 2002. Calciumactivated $\mathrm{K}^{+}$channels of mouse $\beta$-cells are controlled by both store and cytoplasmic $\mathrm{Ca}^{2+}$ : experimental and theoretical studies. J. Gen. Physiol. 114:759769.

Göpel SO, Kanno T, Barg S, Eliasson L, Galvanovskis J, Renström E, Rorsman P. 1999. Activation of $\mathrm{Ca}^{2+}$-dependent $\mathrm{K}^{+}$channels contributes to rhythmic firing of action potentials in mouse pancreatic $\beta$ cells. J. Gen. Physiol. 114:759-769.

Hodgkin AL, Huxley AF. 1952. A quantitative description of membrane current and its application to conduction and excitation in nerve. J Physiol 117(4):500-44.

Hsu H, Huang E, Yang XC, Karschin A, Labarca C, Figl A, Ho B, Davidson N, Lester HA. 1993. Slow and incomplete inactivations of voltage-gated channels dominate encoding in synthetic neurons. Biophys J 65(3):1196-206.

Kahn SE. 2001. Clinical review 135: The importance of beta-cell failure in the development and progression of type 2 diabetes. J Clin Endocrinol Metab 86(9):4047-58.

Keizer J, Magnus G. 1989. The ATP-sensitive potassium channel and bursting in the pancreatic beta cell. Biophys. J. 56:229-242.

Kennedy RT, Kauri LM, Dahlgren GM, Jung S-K. 2002. Metabolic oscillations in $\beta$ cells. Diabetes 51:S152-S161.

Khan FA, Goforth PB, Zhang M, Satin LS. 2001. Insulin activates ATP-sensitive K(+) channels in pancreatic beta-cells through a phosphatidylinositol 3-kinasedependent pathway. Diabetes 50(10):2192-8. 
Martin F, Soria B. 1996. Glucose-induced [Ca2+]i oscillations in single human pancreatic islets. Cell Calcium 20(5):409-14.

Matthews EK, O'Connor MD. 1979. Dynamic oscillations in the membrane potential of pancreatic islet cells. J Exp Biol 81:75-91.

Mears D, Zimliki CL. 2004. Muscarinic agonists activate Ca2+ store-operated and independent ionic currents in insulin-secreting HIT-T15 cells and mouse pancreatic beta-cells. J Membr Biol 197(1):59-70.

Rolland JF, Henquin JC, Gilon P. 2002. G protein-independent activation of an inward $\mathrm{Na}(+)$ current by muscarinic receptors in mouse pancreatic beta-cells. J Biol Chem 277(41):38373-80.

Rorsman P, Renstrom E. 2003. Insulin granule dynamics in pancreatic beta cells. Diabetologia 46(8):1029-45.

Rosario LM, Barbosa RM, Antunes CM, Silva AM, Abrunhosa AJ, Santos RM. 1993. Bursting electrical activity in pancreatic beta-cells: evidence that the channel underlying the burst is sensitive to $\mathrm{Ca} 2+$ influx through L-type $\mathrm{Ca} 2+$ channels. Pflugers Arch 424(5-6):439-47.

Seino S, Miki T. 2003. Physiological and pathophysiological roles of ATP-sensitive K+ channels. Prog Biophys Mol Biol 81(2):133-76.

Sherman A. 1996. Contributions of modeling to understanding stimulus-secretion coupling in pancreatic $\beta$-cells. Am. J. Physiol. 271:E362-E372.

Tengholm A, Hellman B, Gylfe E. 2001. The endoplasmic reticulum is a glucosemodulated high-affinity sink for $\mathrm{Ca} 2+$ in mouse pancreatic beta-cells. J Physiol 530(Pt 3):533-40. 
Tornheim K. 1997. Are metabolic oscillations responsible for normal oscillatory insulin secretion? Diabetes 46:1375-1380.

Unger RH. 2003. Lipid overload and overflow: metabolic trauma and the metabolic syndrome. Trends Endocrinol Metab 14(9):398-403.

Valdeolmillos M, Santos RM, Contreras D, Soria B, Rosario LM. 1989. Glucose-induced oscillations of intracellular $\mathrm{Ca}^{2+}$ concentration resembling electrical activity in single mouse islets of Langerhans. FEBS Lett. 259:19-23.

Wierschem K, Bertram R. 2004. Complex bursting in pancreatic islets: a potential glycolytic mechanism. J Theor Biol 228(4):513-21.

Woods SC, Porte DJ. 1974. Neural control of the endocrine pancreas. Physiol. Rev. 54:596-619.

Worley JF, McIntyre MS, Spencer B, Mertz RJ, Roe MW, Dukes ID. 1994. Endoplasmic reticulum calcium store regulates membrane potential in mouse islet $\beta$-cells. J. Biol. Chem. 269:14359-14362.

\section{Acknowledgements}

This work was supported by NSF grant DMS-0311856 to R. Bertram. 